\title{
Impact of daily Chlorella consumption on serum lipid and carotenoid profiles in mildly hypercholesterolemic adults: a double-blinded, randomized, placebo-controlled study
}

\author{
Na Hee Ryu', Yeni Lim', Ji Eeun Park', Joohee Kim², Ji Yeon Kim³ Sung Won Kwon ${ }^{4^{*}}$ and Oran Kwon ${ }^{1,2,4^{*}}$
}

\begin{abstract}
Background: High level of serum cholesterol is considered to be a major risk factor for cardiovascular disease (CVD). A double-blinded, randomized, placebo-controlled trial was performed to test the hypothesis that a daily intake of Chlorella may improve serum lipid profile through enhancement of serum carotenoid concentration in mildly hypercholesterolemic subjects.
\end{abstract}

Methods: Eligible subjects ( $n=63)$ were randomized to either Chlorella $(5 \mathrm{~g} /$ day) or placebo for a double-blinded trial with a 2-week lead-in period and a 4-week intervention period. Serum triglycerides, total cholesterol, lipoproteins, apolipoproteins and carotenoids were assessed at the beginning and the end of the trial.

Results: Compared with the control group, the Chlorella group exhibited remarkable changes in total cholesterol (Chlorella -1.6\%; placebo 0.03\%; $P=0.036$ ), triglycerides (Ch/orella $-10.3 \%$; placebo 11.9\%; $P=0.002$ ), lutein/zeaxanthin (Chlorella 89.6\%; placebo -1.7\%; $P<0.0001$ ), and a-carotene (Chlorella 163.6\%; placebo 15\%; $P<0.0001$ ). Improvement of serum lipids was supported by significant reductions of very low-density lipoprotein cholesterol (Chlorella $-11 \%$; placebo 11.8\%; $P=0.006)$, apolipoprotein B (Chlorella $-1.5 \%$; placebo 1.7\%; $P=0.044)$, non high-density lipoprotein (Chlorella -2.6\%; placebo -0.5\%; $P=0.032$ ), and high-density lipoprotein/triglycerides (Chlorella 4.0\%; placebo -9.5\%; $P=0.023$ ), suggesting an inhibitory effect of Chlorella on the intestinal absorption of dietary and endogenous lipids. Further, the changes of serum lipids appeared to be associated with the changes of serum carotenoids.

Conclusion: Daily consumption of Chlorella supplements provided the potential of health benefits reducing serum lipid risk factors, mainly triglycerides and total cholesterol, in mildly hypercholesterolemic subjects. The effect was related to carotenoid consumption.

Trial registration: WHO International Clinical Trials Registry Platform KCT0000259.

Keywords: Chlorella, Serum lipid, Carotenoids, Human

\section{Background}

Cardiovascular disease (CVD) is one of the leading causes of death throughout the world. The World Health Organization (WHO) has predicted that the global prevalence of CVD will reach 24 million by 2030 [1]. There is

\footnotetext{
* Correspondence: wizard95@cha.ac.kr; orank@ewha.ac.kr

${ }^{4} \mathrm{CHA}$ Bundang Medical Center, CHA University, Seongnam, Gyeonggi-do 463-712, Republic of Korea

'Department of Nutritional Science and Food Management, Ewha Womans University, Seoul 120-750, Republic of Korea

Full list of author information is available at the end of the article
}

increasing evidence that dietary patterns, certain foods, and food constituents have the potential to benefit CVD prevention through a variety of mechanisms. Total cholesterol (TC) and triglycerides (TG) have featured as important indicators of CVD risk for use in serum [2-5]. Recent data have demonstrated that abnormalities in the metabolism of lipoproteins and apolipoproteins are also responsible for an increased risk of CVDs, supporting the idea that they are more reliable indicator of risk than conventional serum lipid measurements [6,7].

\section{Biomed Central}

(C) 2014 Ryu et al.; licensee BioMed Central Ltd. This is an Open Access article distributed under the terms of the Creative Commons Attribution License (http://creativecommons.org/licenses/by/2.0), which permits unrestricted use, distribution, and reproduction in any medium, provided the original work is properly credited. 
Chlorella is single-celled green alga that is widely marketed as a dietary supplement or incorporated into food. Animal studies demonstrated that Chlorella supplementation reduced the serum cholesterol levels under highfat or high-cholesterol diets [8-10]. Small, open-label trials yielded promising results with a focus on the improvement of serum cholesterol [11-13], but this effect has not been examined in randomized, placebocontrolled trials (RCTs) with a wider array of biomarkers. Chlorella is also known as a potential source of a wide spectrum of nutrients, including chlorophyll, carotenoids, minerals, vitamins, and long-chain polyunsaturated fatty acids [14]. Especially, omega-3 fatty acid and carotenoids have received considerable interests due to their roles in the prevention of chronic diseases and maintaining good health. Many investigators have demonstrated the importance of omega-3 fatty acid for reduction in serum triglyceride concentration [15,16]. The health benefits of carotenoids have been ascribed, in part, to antioxidant scavenging singlet molecular oxygen and peroxyl radicals [17]. Lutein, the most abundant carotenoid in Chlorella, together with zeaxanthin have been shown to be associated with eye health and function [18]. Beta-carotene are known to be inversely related to the risk of CVDs and certain cancers [19]. Alpha and $\beta$-carotene have the added advantage of being able to convert to vitamin $\mathrm{A}$, which is involved in developing and preventing chronic diseases [20]. As for the relationship between carotenoids and cholesterol, most studies have been investigated with respect to the effects of the single carotenoid compound on membrane mechanics [21], intestinal absorption [22] and incorporation into liposomes [23]. To the best of our knowledge, there is no study where the carotenoid in Chlorella has been related to the cholesterol metabolism in humans.

We, therefore, designed a double-blinded RCT to test the hypothesis that a daily intake of Chlorella supplement improves serum lipid profile, which may be attributed to enhancement of serum carotenoids. To this end, we assessed serum TC, TG, lipoproteins, apolipoproteins and carotenoids (lutein/zeaxanthin, $\alpha$-carotene and $\beta$-carotene) in subjects with mild hypercholesterolemia after daily intake of Chlorella supplement for 4 weeks. Moreover, we evaluated the relations between changes in serum lipids and changes in serum carotenoids.

\section{Methods}

\section{Subjects}

One hundred twenty six subjects ( $>20$ years old) were recruited from the CHA Medical Center (Bundang, Gyeonggi-do, Korea). To be eligible, subjects were required to have a serum TC level between 5.18 and $6.48 \mathrm{mmol} / \mathrm{L}$ (mild hypercholesterolemia) at the screening visit. Exclusion criteria were: the consumption of medications and/or dietary supplements that affect lipid metabolisms; pregnancy or lactation; known hypersensitivity to the study product; the presence of familial hypercholesterolemia, CVD, hypertensions, diabetes mellitus, hepatic diseases, kidney diseases, thyroid diseases or other systemic diseases. The study protocol was approved by the Institutional Review Boards of CHA Medical Center and Ewha Womans University (Seoul, Korea).

\section{Test samples}

Tablets composed of Chlorella (Chlorella vulgaris) powder or lactose powder (color-matched placebo) provided by Daesang Corp. (Seoul, Korea) were used in this trial. The Chlorella tablet $(650 \mathrm{mg})$ contained $416 \mathrm{mg}$ of Chlorella powder with the following nutritional composition (per $100 \mathrm{~g}$ ): $5.4 \mathrm{~g}$ moisture, $60.6 \mathrm{~g}$ protein, $3.7 \mathrm{~g}$ total carbohydrates, $12.8 \mathrm{~g}$ total lipids, $13.0 \mathrm{~g}$ dietary fiber, $4.5 \mathrm{~g}$ ash, 58,900 IU vitamin A, $74 \mathrm{mg}$ vitamin C, $22.8 \mathrm{mg}$ vitamin $\mathrm{E}$, and $2.4 \mathrm{~g}$ chlorophylls [10]. The carotenoid content of Chlorella powder was determined in our laboratory by using a high-performance liquid chromatography (HPLC) method [24] and the results are as follows (per $100 \mathrm{~g}$ ): $260 \mathrm{mg}$ lutein, $5 \mathrm{mg}$ zeaxanthin, $24 \mathrm{mg} \alpha$-carotene, and $17 \mathrm{mg} \beta$-carotene.

\section{Study design}

After initial screening and the following 2-week lead-in phase, 68 eligible subjects ( 44 female and 19 male) were randomized into either Chlorella or placebo in a doubleblinded fashion. The participants were advised to maintain their usual level of activity and diet, but to avoid certain high-cholesterol and high-carotenoid foods during the lead-in and the intervention days. They were also advised to take 4 tablets of test sample with water immediately after each meal, a total of 12 tablets ( $5 \mathrm{~g}$ as Chlorella powder) a day, for 4 weeks. To monitor the subjects' dietary compliances and assess their nutrient intake, three-day food diaries (including two weekdays and one weekend day) were completed at the beginning and end of the study. Energy and nutrient intakes were calculated using a computer-aided nutritional analysis program (CAN-pro 3.0, Korean Nutrition Society, Seoul, Korea).

\section{Measurements}

Following completion of the lead-in phase and the 4-week intervention period, overnight fasting venous blood was collected from a vein on the flexor side of the arm into a serum separator tubes (BD Biosciences, San Jose, CA, USA). The concentrations of TC, TG, low-density lipoprotein-cholesterol (LDL-C), and highdensity lipoprotein-cholesterol (HDL-C) were simultaneously measured using an automatic analyzer (Hitachi 7600, Hitachi High-Technologies Co., Osaka, Japan). Very low-density lipoprotein-cholesterol (VLDL-C) and 
non-HDL-C were calculated as TG/2.2 and the difference between TC and HDL-C, respectively [25]. Serum apolipoproteins (apo A1, apo B and apo E) were measured using a BNII nephelometer (Siemens, Munich, Germany). Serum carotenoids were analyzed after deproteinizing with ethanol containing the internal standard ( $\alpha$-tocopherol acetate) and then extracting with hexane. After evaporation, the residue was dissolved in mobile phase and quantified by using HPLC (Shiseido SP 3023) [24].

\section{Statistics}

We estimated the sample size needed to detect between group differences on serum TC at the end of 4-week intervention period to be 34 subjects per group. With these estimates and a 2 -sided $\alpha=0.05$, the study had greater than $80 \%$ power with up to $10 \%$ dropout. The statistical analyses were performed with the SAS program package, version 9.3 (SAS Institute, Cary, NC, USA). The normal distribution of each variable was tested before statistical testing and logarithmic transformation was performed on skewed variables. Baseline comparisons between groups were determined using Student's $t$-test for independent samples. Differences in means for the efficacy of Chlorella versus placebo were analyzed by a one-way analysis of covariance (ANCOVA) after adjusting for the baseline values as covariates. Differences in means for absolute change from baseline to 4-week within groups were evaluated using paired $t$-tests. Pearson's correlation was used to determine the strength of the relationships between the serum lipids and the serum carotenoids for all participants, irrespective of treatment group. For descriptive purposes, mean values of untransformed and unadjusted variables are presented. A two-tailed value of $P<0.05$ was considered statistically significant.

\section{Results}

\section{Subject characteristics and diet monitoring}

Of the 68 participants who commenced the study, 63 completed the endpoint assessments (Chlorella, $n=33$; placebo, $n=30$ ). Five participants (4 in the placebo group and 1 in the Chlorella group) withdrew during the intervention due to either protocol deviations $(n=3)$ or consent withdrawals $(n=2)$ (Figure 1). No serious adverse events or side effects were observed during the intervention period.

The participants represented a mildly hypercholesterolemic population with a mean level of $5.64 \pm 0.07 \mathrm{mmol} / \mathrm{L}$ TC, $3.26 \pm 0.07 \mathrm{mmol} / \mathrm{L} \mathrm{LDL}-\mathrm{C}$, and $1.43 \pm 0.05 \mathrm{mmol} / \mathrm{L}$ HDL-C. The randomization was successful; the two groups did not differ significantly at baseline in terms of the measured endpoints (Table 1). Furthermore, the data from the dietary records indicated that the participants generally consumed diets with similar levels of energy intake, carbohydrates, proteins, fats and cholesterol at baseline and during the study. Carotenoid intake increased significantly by supplementing $5 \mathrm{~g}$ of Chlorella as compared with placebo $(P=0.0003)$ (Table 2$)$.

\section{Serum lipid and carotenoid profiles}

While no significant changes were observed in the placebo group, the Chlorella group showed significant reductions from baseline in $\mathrm{TC}(1.6 \%$ reduction, $P=0.046)$, TG $(10.3 \%$ reduction, $P=0.029)$, VLDL-C (11\% reduction, $P=0.029)$, non-HDL-C (2.6\% reduction, $P=0.024)$, and HDL-C/TG (4.0\% increase. $P=0.035)$. The differences in means between groups were also significant for TC $(P=0.036)$, TG $(P=0.002)$, VLDL-C $(P=0.032)$, non-HDL-C $(P=0.006)$, and HDL-C/TG $(P=0.023)$, indicating true supplement effects (Figure $2 \mathrm{~A})$. The serum LDL-C and HDL-C levels, however, did not change significantly in the Chlorella group during the study; thus, there were no significant differences between the groups. Apolipoproteins were also measured, showing a significant decrease of apo B concentration in the Chlorella group $(P=0.129)$ and increased in the control group $(P=0.113)$, resulting in a significant difference between the two groups $(P=0.044)$. However, there were no significant differences in apo A1 and apo E between the two groups (Figure 2B).

The changes in serum carotenoids were shown in Figure 3. As predicted based on the carotenoid composition of Chlorella, the serum lutein/zeaxanthin and $\alpha$-carotene levels were significantly increased followed by Chlorella supplementation within groups $(P<0.0001)$ and compared with the control group $(P<0.0001)$. Unexpectedly serum $\beta$-carotene concentrations exhibited a significant decrease from baseline value in the placebo group compared with the Chlorella group $(P=0.043)$, resulting in a significant difference between the two groups $(P=0.029)$.

\section{Correlation between changes in serum lipids and carotenoids}

The analysis of correlation revealed that the serum TG concentrations showed a tendency toward a negative correlation with the serum lutein/zeaxanthin $(r=-0.25$, $P=0.052)$ and $\alpha$-carotene $(r=-0.23, P=0.074)$ for all groups (Figure 4A). However, a contrary effect was found in the relationship between the serum TC and the serum lutein/zeaxanthin $(r=0.31, P=0.013)$. No correlation was found between the serum TC and the serum $\alpha$-carotene (Figure 4B).

\section{Discussion}

To the best of our knowledge, the present study is the first evidence offered to support the hypothesis that the 


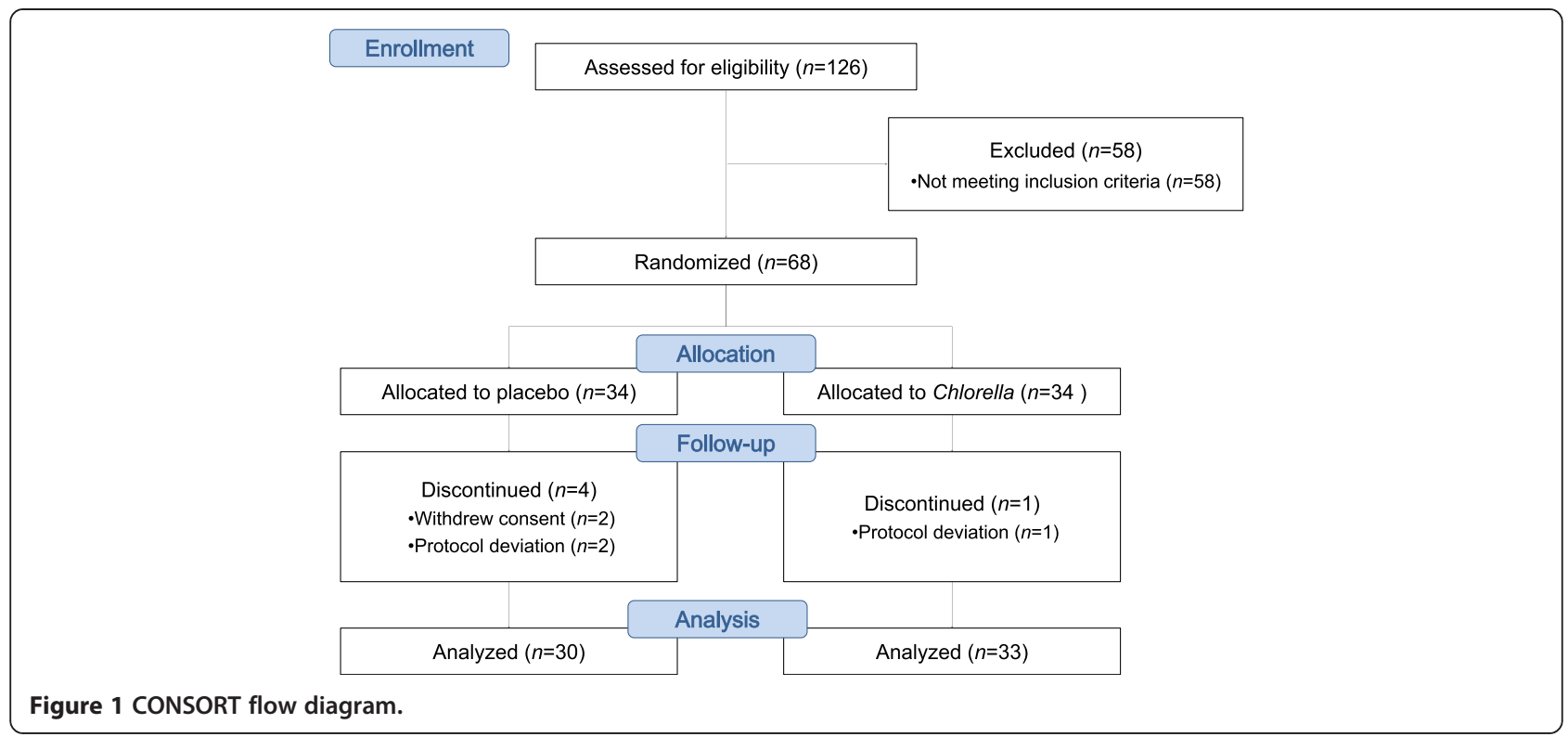

regular consumptions of Chlorella supplement ( $5 \mathrm{~g} /$ day) over 4 weeks significantly reduced serum TG, TC, non-HDL-C, VLDL-C, HDL-C/TG, and apo B in subjects with mild hypercholesterolemia using RCT study design. This study also demonstrated that daily consumption of Chlorella supplement resulted in significant increases inserum lutein/zeaxanthin and $\alpha$-carotene concentrations. In addition, the changes of serum TG and TC appeared to be associated with the changes in serum lutein/zeaxanthin and $\alpha$-carotene.
Some studies in open-label ("before and after") design with relatively small numbers of subjects have shown the effect of Chlorella, focusing on the serum cholesterol levels rather than overall lipid and carotenoid profiles. Okuda et al. [11] showed that the daily intakes of $5 \mathrm{~g}$ of Chlorella for three months significantly lowered the TC of subjects with hypercholesterolemia $(n=16)$ to almost the normal level. In another study, Youko et al. [12] demonstrated reductions in TC and LDL-C concentrations after

Table 1 Baseline characteristics of subjects who completed the intervention trial

\begin{tabular}{|c|c|c|c|}
\hline Variables & Control $(n=30)$ & Chlorella $(n=33)$ & $P$-value \\
\hline Age & $50.9 \pm 1.6$ & $48.2 \pm 1.4$ & 0.281 \\
\hline Female/male (n) & $22 / 8$ & $22 / 11$ & 0.565 \\
\hline $\mathrm{BMI}\left(\mathrm{kg} / \mathrm{m}^{2}\right)$ & $24.5 \pm 0.5$ & $23.9 \pm 0.6$ & 0.488 \\
\hline Fasting glucose $(\mathrm{mmol} / \mathrm{L})^{\#}$ & $5.5 \pm 0.1$ & $5.6 \pm 0.1$ & 0.499 \\
\hline Systolic BP (mmHg) & $120.1 \pm 2.1$ & $120.0 \pm 2.0$ & 0.981 \\
\hline Diastolic BP (mmHg) & $75.8 \pm 1.3$ & $76.4 \pm 1.7$ & 0.787 \\
\hline \multicolumn{4}{|l|}{ Lipid profiles } \\
\hline $\mathrm{TC}(\mathrm{mmol} / \mathrm{L})$ & $5.68 \pm 0.10$ & $5.60 \pm 0.10$ & 0.587 \\
\hline $\mathrm{TG}(\mathrm{mmol} / \mathrm{L})^{\#}$ & $1.49 \pm 0.14$ & $1.90 \pm 0.23$ & 0.137 \\
\hline $\mathrm{LDL}-\mathrm{C}(\mathrm{mmol} / \mathrm{L})$ & $3.34 \pm 0.10$ & $3.18 \pm 0.09$ & 0.262 \\
\hline $\mathrm{HDL}-\mathrm{C}(\mathrm{mmol} / \mathrm{L})$ & $1.40 \pm 0.06$ & $1.43 \pm 0.06$ & 0.914 \\
\hline VLDL-C $(\mathrm{mmol} / \mathrm{L})^{\#}$ & $0.68 \pm 0.06$ & $0.86 \pm 0.11$ & 0.163 \\
\hline non-HDL-C (mmol/L) & $4.26 \pm 0.11$ & $4.17 \pm 0.11$ & 0.568 \\
\hline Apo A1 (g/L) & $1.47 \pm 0.05$ & $1.52 \pm 0.05$ & 0.493 \\
\hline Apo B (g/L) & $1.07 \pm 0.03$ & $1.08 \pm 0.02$ & 0.886 \\
\hline Apo $E(g / L)^{\#}$ & $0.05 \pm 0.00$ & $0.06 \pm 0.00$ & 0.747 \\
\hline
\end{tabular}

All values are means \pm SEM. Student's $t$-test or chi-square test was used to analyze the data. $B M I$ body mass index; $B P$ blood pressure; $T C$ total cholesterol; $T G$ triglycerides; $L D L-C$ low-density lipoprotein cholesterol; $H D L-C$ high-density lipoprotein cholesterol; VLDL-C very low-density lipoprotein cholesterol; Apo apo-lipoprotein.

\#Tested after log transformation. 
Table 2 Mean daily intake of energy and selected nutrients at baseline and after intervention

\begin{tabular}{|c|c|c|c|c|c|c|}
\hline \multirow[t]{2}{*}{ Nutrients } & \multicolumn{2}{|c|}{ Placebo $(n=30)$} & \multicolumn{2}{|c|}{ Chlorella $(n=33)$} & \multicolumn{2}{|l|}{$P$-value ${ }^{\S}$} \\
\hline & Week 0 & Week 4 & Week 0 & Week 4 & Week 0 & Week 4 \\
\hline Energy (kcal) & $1527 \pm 71$ & $1560 \pm 66$ & $1695 \pm 85$ & $1664 \pm 66$ & 0.135 & 0.270 \\
\hline Carbohydrate (g) & $234.8 \pm 10.0$ & $240.2 \pm 8.0$ & $250.7 \pm 11.5$ & $247.1 \pm 9.7$ & 0.304 & 0.591 \\
\hline Dietary fibers (g) & $19.9 \pm 1.0$ & $22.6 \pm 1.1^{*}$ & $19.7 \pm 1.1$ & $21.6 \pm 1.2^{*}$ & 0.900 & 0.539 \\
\hline Protein (g) & $61.5 \pm 3.1$ & $62.5 \pm 3.2$ & $69.1 \pm 4.2$ & $67.5 \pm 3.1$ & 0.183 & 0.280 \\
\hline Total fat (g) & $38.8 \pm 2.5$ & $38.9 \pm 2.7$ & $45.9 \pm 3.4$ & $45.4 \pm 2.8$ & 0.114 & 0.108 \\
\hline SFA (g) & $5.7 \pm 0.5$ & $6.0 \pm 0.8$ & $7.2 \pm 0.9$ & $6.4 \pm 0.6$ & 0.346 & 0.480 \\
\hline MUFA (g) & $7.1 \pm 0.6$ & $7.3 \pm 0.9$ & $8.4 \pm 1.0$ & $8.2 \pm 0.7$ & 0.457 & 0.273 \\
\hline PUFA (g) & $6.1 \pm 0.6$ & $6.4 \pm 0.6$ & $7.0 \pm 0.6$ & $6.2 \pm 0.4$ & 0.260 & 0.854 \\
\hline Cholesterol (mg) & $271.9 \pm 26.6$ & $289.3 \pm 27.8$ & $303.2 \pm 27.7$ & $269.2 \pm 21.3$ & 0.339 & 0.564 \\
\hline$\beta$-carotene $(\mathrm{mg})^{\#}$ & $2.8 \pm 0.2$ & $3.1 \pm 0.3$ & $3.0 \pm 0.4$ & $4.7 \pm 0.4^{*}$ & 0.711 & 0.0003 \\
\hline
\end{tabular}

All values are means \pm SEM. Intakes were estimated from three-day food records by using CAN-pro (Korean Nutrition Society, Seoul, Korea). Intakes of test products were included in the analysis. SFA saturated fatty acids; MUFA monounsaturated fatty acids; PUFA polyunsaturated fatty acids.

\#Tested after log transformation.

*Significantly different within a group, $P<0.05$ (paired $t$-test).

${ }^{\S}$ Comparison between the placebo and the Chlorella group at week 0 and week 4, respectively (Student's $t$-test).

$9 \mathrm{~g}$ of Chlorella supplementation for 12 months in patients with hyperlipidemia $(n=9)$. Sansawa et al. [13] found that the daily ingestion of $3 \mathrm{~g}$ of Chlorella for three months lowered serum TC and LDL-C in patients with mild hypercholesterolemia $(n=20)$. These small studies suggested a potential of Chlorella supplementation for reductions of TC at doses of 3-9 g, providing a rationale for further evaluations of Chlorella in a randomized, placebocontrolled trials.

In the lipoprotein lipolytic cascade, apo B is required for the secretion of VLDL from the liver and converted to intermediate-density lipoprotein (IDL) by lipoprotein lipase in the endothelial surface of capillaries. The IDL particles can be rapidly taken up and then catabolized mainly in the liver via apo E, a high-affinity ligand for the receptor. However, some IDL particles escape hepatic uptake and remain in the circulation due to lack of apo E and are further converted to LDL [26]. The binding affinity of apo B was relatively low, and thus, LDL particles circulated for a relatively prolonged period before binding to LDL receptors throughout the body $[27,28]$. There is a systematically only one apo B per LDL particle and thus plasma level of apo B is strongly associated with LDL particle number, which is an important predictor of cardiovascular risks [29]. Conversely, the HDL accepts cholesterol from peripheral cells and transports it to the liver for bile production. Apo A1 is the major protein constituent of HDL particles, mediating reverse cholesterol transport [30]. It is important to note that the most pronounced effect of Chlorella in the current study were decreases in VLDL and apo $\mathrm{B}$ concentrations. This result implicates that there are slowed secretion of apo B and VLDL into the circulation or faster catabolism of VLDL in the liver.

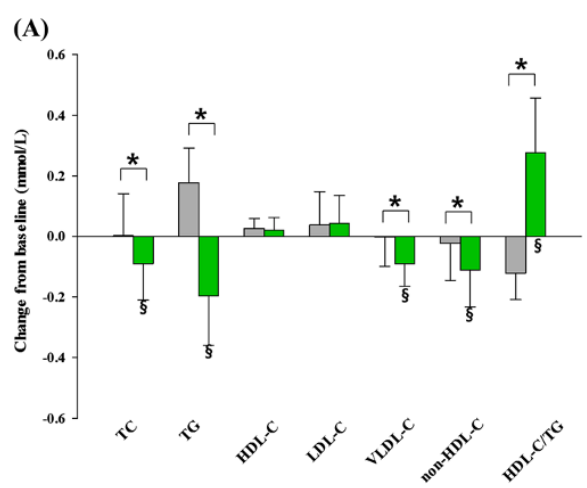

(B)

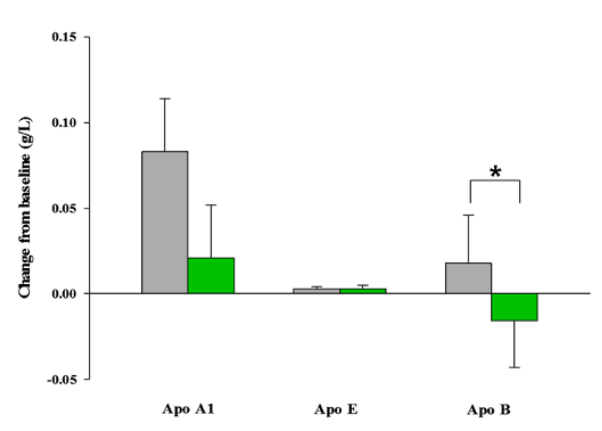

Figure 2 Mean changes of serum lipids profile in subjects with mild hypercholesterolemia. Panel $\mathbf{A}$ shows the changes of lipids and lipoproteins for Chlorella (green rectangle, $n=33$ ) and placebo (gray rectangle, $n=30$ ). Panel $\mathbf{B}$ shows the changes of apolipoproteins. The data represent means \pm SEM. ${ }^{*} P<0.05$ compared between groups using ANCOVA after adjusting for the baseline value. ${ }^{\S} P<0.05$ compared to baseline using paired $t$-test. 


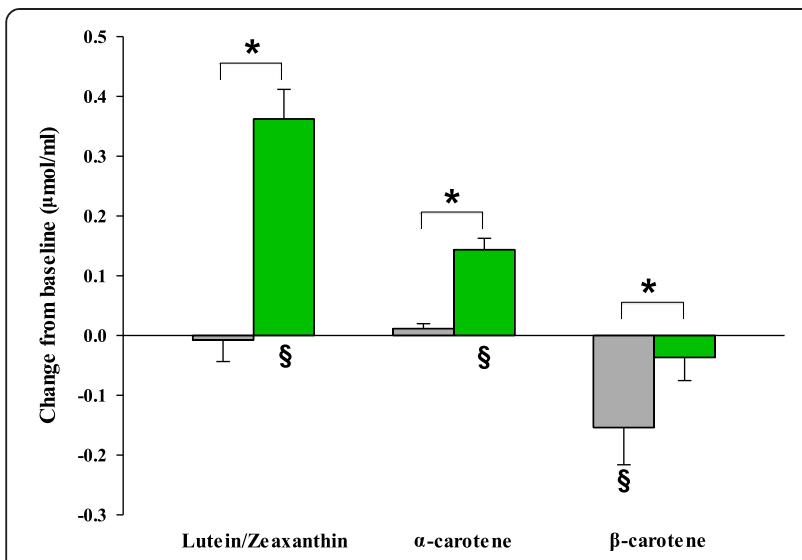

Figure 3 Mean changes of serum carotenoids profile in subjects with mild hypercholesterolemia. Chlorella (green rectangle, $n=33$ ) and placebo (gray rectangle, $n=30$ ). The data represent means \pm SEM. ${ }^{*} P<0.05$ compared between groups using ANCOVA after adjusting for the baseline value. ${ }^{\S} P<0.05$ compared to baseline using paired $t$-test.

This is speculative but the first part is consistent with the previous findings that Chlorella plays an important role in inhibiting the intestinal absorptions of dietary lipids in animals [8-10]. In addition, lowering apo B compromises lowering LDL particle number and promotes CVD risk. It is also worth noting that, in the present study, four weeks of Chlorella supplementation produced no noticeable effects on HDL-C, apo E, and apo A1 compared with the control group, implicating that reverse cholesterol transport was unaffected by Chlorella consumption. The design of the current study did not allow the direct determination of cholesterol absorption. Considering all the results together, however, we can assume that the inhibition of intestinal lipid absorption may have caused the serum lipid profile changes observed in the Chlorella group.

Chlorella provides a wide range of nutrients and phytochemicals that work synergistically to optimize lipid metabolism. It contains a relatively high percentage of omega-3 fatty acids [14], which are known not to inhibit chylomicron assembly in the intestine, but to inhibit VLDL assembly in the liver [15]. Chlorella is a good source of dietary fiber that affects lymphatic cholesterol and triglyceride absorption by increasing gut viscosity, altering the composition of the bile acid pool, or producing fermentation products in the intestine [31]. Chlorella is also a good source of carotenoids and it significantly increased serum concentration of lutein/zeaxanthin and $\alpha$-carotene. The steps of carotenoid absorption are similar to those for dietary lipids: the release from the food matrix, solubilization in mixed micelles, packaging into chylomicrons, and secretion into the lymphatic system [32]. Therefore, one can assume that the presence of increased concentrations of carotenoids released from Chlorella compete with dietary lipids for incorporation and transport in lipoproteins, therefore causing the decreased serum lipid levels. In a previous animal study Nicolle et al. [33] investigated the effects of carrot supplementation on lipid metabolism in rats, suggesting that dietary carotenoid consumption modifies lipid absorptions. In this study, we found that individuals with higher levels of serum lutein/zeaxanthin and $\alpha$-carotene had greater potentials to reduce serum TG concentrations, supporting the notion that carotenoids may inhibit TG absorption. However, contrary to TG, no or weak positive correlation was found between the changes in serum carotenoids and the changes in TC. One possible explanation for these conflict may be that
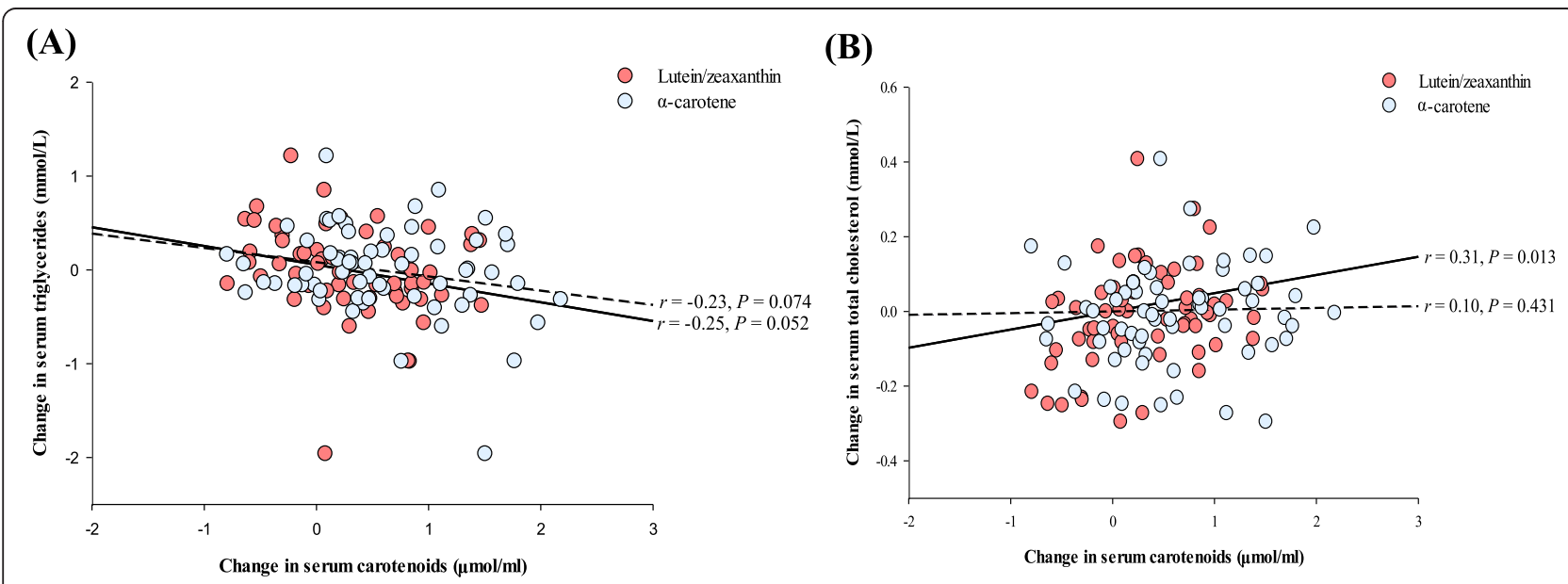

Figure 4 Correlation between the changes of serum carotenoids and lipids. Panel $\mathbf{A}$ shows a correlation between lutein/zeaxanthin or a-carotene and TG for all subjects $(n=63)$. Panel B shows the same analysis for TC. The Pearson correlation $r$ - and $P$-values for each curve are shown adjacent to the line in the respective panel. 
the polar carotenoids are mostly distributed in association with cholesterol-rich lipoproteins in the serum [32], which masks the degree of inhibitory effects of carotenoids on cholesterol absorption. This notion is further supported by the results of Loane et al. [34], who reported a positive association between serum lutein concentration and both serum LDL and HDL level among healthy subjects.

There are several limitations in this study. First, the suggested molecular mechanism by which Chlorella are thought to exert their effect was not directly determined. Despite this limitation, however, our findings raised the possibility that the effects of Chlorella on the inhibition of intestinal absorption of both dietary and endogenous lipids may have caused the serum lipid profile changes. Another limitation of this study is that there was no more biochemical data to show the localization of carotenoids in lipoproteins. Future studies are warranted to prove these initial findings.

\section{Conclusions}

The present study revealed that Chlorella appears to have beneficial health effects on the serum lipid profiles of mildly hypercholesterolemic subjects at least in part via improvement of serum carotenoid profiles. Our findings also suggest that possible effects of Chlorella on serum lipids might be caused by a suppression of intestinal lipid absorption attributable to increased levels of highly polar carotenoids from Chlorella. Although further studies are required to determine the direct effects of Chlorella for the absorptions of dietary lipids, the results of this study provide evidence to previous observations, which suggest that Chlorella should be included on the list for recommending heart-healthy dietary supplements.

\section{Abbreviations \\ CVD: Cardiovascular disease; TC: Total cholesterol; TG: Triglycerides; \\ RCT: Randomized-placebo-controlled trial; VLDL: Very low-density lipoprotein;} LDL: Low-density lipoprotein; HDL: High-density lipoprotein.

\section{Competing interests}

The authors have no conflict of interest.

\section{Authors' contribution}

OK, SWK, JYK and JK were responsible for conception and study design; OK and SWK were responsible for the supervision of the human study, sample handling, conduction of the analyses, and statistical analysis; NHR and JEP conducted the study and performed analyses of the data; $Y L$ and JK performed the statistical analysis; $Y L$ and OK prepared the manuscript; all authors read and approved the final manuscript.

\section{Acknowledgments}

This study was supported by Daesang Corp. (Seoul, Korea) and the Ministry of Science, ICT \& Future Planning (NRF 2013M3A9C4078153) and the Ministry of Education, Science and Technology (BK21 PLUS 22A20130012143). The funding sources had no involvement in the study design; in the collection, analysis, and interpretation of the data; in the writing of this report; or in the decision to submit it for publication. The authors are grateful to the volunteers for taking part in this study.

\section{Author details}

${ }^{1}$ Department of Nutritional Science and Food Management, Ewha Womans University, Seoul 120-750, Republic of Korea. ${ }^{2}$ BioFood Network, Ewha Womans University, Seoul 120-750, Republic of Korea. ${ }^{3}$ Department of Food Science and Technology, Seoul National University of Science and Technology, Seoul 139-743, Republic of Korea. ${ }^{4} \mathrm{CHA}$ Bundang Medical Center, CHA University, Seongnam, Gyeonggi-do 463-712, Republic of Korea.

Received: 2 September 2013 Accepted: 14 May 2014

Published: 11 June 2014

\section{References}

1. Lloyd-Jones D, Adams R, Carnethon M, De Simone G, Ferguson TB, Flegal K, Ford E, Furie K, Go A, Greenlund K, Hasse N, Hailpern S, Ho M, Howard V, Kissela B, Kittner S, Lackland D, Lisabeth L, Marelli A, McDermott M, Meigs J, Mozaffarian D, Nichol G, O'Donnell C, Roger V, Rosamond W, Sacco R, Sorlie P, Stafford R, Steinberger J, et al: Heart disease and stroke statistics-2009 update: a report from the American Heart Association Statistics Committee and Stroke Statistics Subcommittee. Circulation 2009, 119:e21-e181.

2. McPherson R, Frohlich J, Fodor G, Genest J, Canadian Cardiovascular Society: Canadian Cardiovascular Society position statement-recommendations for the diagnosis and treatment of dyslipidemia and prevention of cardiovascular disease. Can J Cardiol 2006, 22:913-927.

3. Mora S, Otvos JD, Rifai N, Rosenson RS, Buring JE, Ridker PM: Lipoprotein particle profiles by nuclear magnetic resonance compared with standard lipids and apolipoproteins in predicting incident cardiovascular disease in women. Circulation 2009, 119:931-939.

4. Nordestgaard BG, Benn M, Schnohr P, Tybjaerg-Hansen A: Nonfasting triglycerides and risk of myocardial infarction, ischemic heart disease, and death in men and women. JAMA 2007, 298:299-308.

5. Sarwar N, Sandhu MS, Ricketts SL, Butterworth AS, Di Angelantonio E, Boekholdt SM, Ouwehand W, Watkins H, Samani NJ, Saleheen D, Lawlor D, Reilly MP, Hingorani AD, Talmud PJ, Danesh J: Triglyceride-mediated pathways and coronary disease: collaborative analysis of 101 studies. Lancet 2010, 375:1634-1639.

6. Sacks FM, Alaupovic P, Moye LA, Cole TG, Sussex B, Stampfer MJ, Pfeffer MA, Braunwald E: VLDL, apolipoproteins $B, C I I I$, and $E$, and risk of recurrent coronary events in the Cholesterol and Recurrent Events (CARE) trial. Circulation 2000, 102:1886-1892.

7. Contois JH, McConnell JP, Sethi AA, Csako G, Devaraj S, Hoefner DM, Warnick GR, Practices ALaVDDWGoB: Apolipoprotein B and cardiovascular disease risk: position statement from the AACC lipoproteins and vascular diseases division working group on best practices. Clin Chem 2009, 55:407-419.

8. Sano T, Tanaka Y: Effect of dried, powdered Chlorella vulgaris on experimental atherosclerosis and alimentary hypercholesterolemia in cholesterol-fed rabbits. Artery 1987, 14:76-84.

9. Shibata S, Oda K, Onodera-Masuoka N, Matsubara S, Kikuchi-Hayakawa H, Ishikawa F, Iwabuchi A, Sansawa H: Hypocholesterolemic effect of indigestible fraction of Chlorella regularis in cholesterol-fed rats. J Nutr Sci Vitaminol (Tokyo) 2001, 47:373-377.

10. Lee HS, Park HJ, Kim MK: Effect of Chlorella vulgaris on lipid metabolism in Wistar rats fed high fat diet. Nutr Res Pract 2008, 2:204-210.

11. Masso O, Hasegawa T, Sonoda M, Okabe T, Tanaka Y: The effects of Chlorella on the levels of cholesterol in serum and liver. Jap J Nutr 1975, 33:8.

12. Fujiwara Y, Hirakawa K, Kunihiro S: Effect of Long-term administration of chlorella tablets on hyperlipemia. J Jpn Soc Nutr F Sci 1990, 43:167-173.

13. Sansawa H, Inoue $K$, Shirai T: Effect of Chlorella tablet ingestion on mild hypercholesterolemic patients. Nippon Shokuhin Kagaku Kaishi 2002, 49:6

14. Bocanegra A, Bastida S, Benedí J, Ródenas S, Sánchez-Muniz FJ: Characteristics and nutritional and cardiovascular-health properties of seaweeds. J Med Food 2009, 12:236-258.

15. Park Y, Harris WS: Omega-3 fatty acid supplementation accelerates chylomicron triglyceride clearance. J Lipid Res 2003, 44:455-463.

16. Connor WE: Importance of $\mathrm{n}-3$ fatty acids in health and disease. Am J Clin Nutr 2000, 71:171S-175S.

17. Christaki E, Florou-Paneri $P$, Bonos E: Microalgae: a novel ingredient in nutrition. Int J Food Sci Nutr 2011, 62:794-799. 
18. Richer S, Stiles W, Statkute L, Pulido J, Frankowski J, Rudy D, Pei K, Tsipursky M, Nyland J: Double-masked, placebo-controlled, randomized trial of lutein and antioxidant supplementation in the intervention of atrophic age-related macular degeneration: the Veterans LAST study (Lutein Antioxidant Supplementation Trial). Optometry 2004, 75:216-230.

19. Johnson EJ: The role of carotenoids in human health. Nutr Clin Care 2002, 5:56-65.

20. Rao AV, Rao LG: Carotenoids and human health. Pharmacol Res 2007, 55:207-216

21. Socaciu C, Bojarski P, Aberle L, Diehl H: Different ways to insert carotenoids into liposomes affect structure and dynamics of the bilayer differently. Biophys Chem 2002, 99:1-15.

22. During A, Dawson HD, Harrison EH: Carotenoid transport is decreased and expression of the lipid transporters SR-BI, NPC1L1, and ABCA1 is downregulated in Caco-2 cells treated with ezetimibe. J Nutr 2005, 135:2305-2312

23. Socaciu C, Jessel R, Diehl HA: Competitive carotenoid and cholesterol incorporation into liposomes: effects on membrane phase transition, fluidity, polarity and anisotropy. Chem Phys Lipids 2000, 106:79-88.

24. Zonta F, Stancher B, Marletta GP: Simultaneous high-performance liquid chromatographic analysis of free carotenoids and carotenoid esters. J Chromatogr 1987, 403:207-215.

25. Friedewald WT, Levy RI, Fredrickson DS: Estimation of the concentration of low-density lipoprotein cholesterol in plasma, without use of the preparative ultracentrifuge. Clin Chem 1972, 18:499-502.

26. Kita T, Brown MS, Bilheimer DW, Goldstein JL: Delayed clearance of very low density and intermediate density lipoproteins with enhanced conversion to low density lipoprotein in WHHL rabbits. Proc Natl Acad Sci U S A 1982, 79:5693-5697.

27. Packard CJ, Demant T, Stewart JP, Bedford D, Caslake MJ, Schwertfeger G, Bedynek A, Shepherd J, Seidel D: Apolipoprotein B metabolism and the distribution of VLDL and LDL subfractions. J Lipid Res 2000, 41:305-318.

28. Ishibashi S, Brown MS, Goldstein JL, Gerard RD, Hammer RE, Herz J: Hypercholesterolemia in low density lipoprotein receptor knockout mice and its reversal by adenovirus-mediated gene delivery. J Clin Invest 1993, 92:883-893.

29. Lamarche B, Tchernof A, Moorjani S, Cantin B, Dagenais GR, Lupien PJ, Després JP: Small, dense low-density lipoprotein particles as a predictor of the risk of ischemic heart disease in men. Prospective results from the Québec Cardiovascular Study. Circulation 1997, 95:69-75.

30. Borecki IB, Laskarzewski P, Rao DC: Genetic factors influencing apolipoprotein Al and All levels in a kindred with premature coronary heart disease. Genet Epidemiol 1988, 5:393-406.

31. Queenan KM, Stewart ML, Smith KN, Thomas W, Fulcher RG, Slavin JL: Concentrated oat beta-glucan, a fermentable fiber, lowers serum cholesterol in hypercholesterolemic adults in a randomized controlled trial. Nutr J 2007, 6:6.

32. Erdman JW, Bierer TL, Gugger ET: Absorption and transport of carotenoids. Ann N Y Acad Sci 1993, 691:76-85.

33. Nicolle C, Cardinault N, Aprikian O, Busserolles J, Grolier P, Rock E, Demigné C, Mazur A, Scalbert A, Amouroux P, Rémésy C: Effect of carrot intake on cholesterol metabolism and on antioxidant status in cholesterol-fed rat. Eur J Nutr 2003, 42:254-261.

34. Loane E, Nolan JM, Beatty S: The respective relationships between lipoprotein profile, macular pigment optical density, and serum concentrations of lutein and zeaxanthin. Invest Ophthalmol Vis Sci 2010, 51:5897-5905.

doi:10.1186/1475-2891-13-57

Cite this article as: Ryu et al: Impact of daily Chlorella consumption on serum lipid and carotenoid profiles in mildly hypercholesterolemic adults: a double-blinded, randomized, placebo-controlled study. Nutrition Journal 2014 13:57.

\section{Submit your next manuscript to BioMed Central and take full advantage of:}

- Convenient online submission

- Thorough peer review

- No space constraints or color figure charges

- Immediate publication on acceptance

- Inclusion in PubMed, CAS, Scopus and Google Scholar

- Research which is freely available for redistribution

Submit your manuscript at www.biomedcentral.com/submit 